\title{
Three-Level $\Lambda$-Type Atomic System Localized by the Parameters of the Two Orthogonal Standing-Wave Fields
}

\author{
Shuncai Zhao', Qixuan $\mathrm{Wu}^{2}$ \\ ${ }^{1}$ Department of Physics, Faculty of Science, Kunming University of Science and Technology, Kunming, China \\ ${ }^{2}$ College English Department, Kunming University of Science and Technology, Kunming, China \\ Email: zscnum1@126.com, zscgroup@kmust.edu.cn
}

Received 19 July 2016; accepted 14 August 2016; published 17 August 2016

Copyright (C) 2016 by authors and Scientific Research Publishing Inc.

This work is licensed under the Creative Commons Attribution International License (CC BY). http://creativecommons.org/licenses/by/4.0/

\section{(c) (i) Open Access}

\begin{abstract}
Localization of the three-level $\Lambda$-type atomic system interacting with two orthogonal standingwave fields is proposed. Two equal and tunable peaks in the $2 \mathrm{D}$ plane are obtained by the detunings corresponding to the two orthogonal standing-wave fields when the decreasing intensities of spontaneously generated coherence (SGC) arise in the three-level $\Lambda$-type atomic system, while one circular ring with shrinking radii in the $2 \mathrm{D}$ plane is obtained by the adjusted phases and wave vectors of the standing-wave fields when the increasing intensities of SGC occur in the three-level $\Lambda$-type atomic system. 2D atom localization with the single ring with shrinking radii realized by the multiple parametric manipulations demonstrated the flexibility for our scheme.
\end{abstract}

\section{Keywords}

Three-Level $\Lambda$-Type Atomic System, Atom Localization, Spontaneously Generated Coherence (SGC)

\section{Introduction}

Because precise position measurement of a single atom has some potential applications, such as laser cooling and trapping of neutral atoms [1] [2], atom nanolithography [3], Bose-Einstein condensation [4], and measurement of center-of-mass wave function of moving atoms [5] [6], etc., atomic localization has attracted considerable investigation. Utilizing the interacting between an atom and the standing-wave field, one dimensional (1D) atom localization within the optical wavelength domain is realized by the measurement of the phase shift [7] [8], homodyne detection [9], quantum trajectories [10], the dual quadrature field [11], the upper level population [12] 
[13], the probe field absorption or gain [14]-[16], two-photon spontaneous emission [17], the coherent population trapping [18] and the reservoir modes [19]-[21], etc.

More recently, the researchers proposed two-dimensional (2D) atom localization schemes [22]-[26], in which the two orthogonal standing-wave fields are employed. The 2D atom localization was obtained via measurement of the population in the upper or any ground state in a four-level tripod system [22], in which some interesting spatial periodic structures, such as spikes, craters, and waves are observed. Wan et al. suggested the 2D atom localization scheme via incorporating the quantum interference phenomenon in a coherently driven inverted-Y system [23]. Recently, some other schemes, such as via spontaneous emission in a coherently driven five-level M-type atomic system [24], and via the probe absorption in microwave-driven atomic system [25] [26] have been proposed for 2D atom localization.

On the other hand, the phrase of SGC is a well-known concept in quantum optics, which refers to the interference of spontaneous emission channels [27] firstly suggested by Agarwal [28] who showed that the spontaneous emission from a degenerate $\Lambda$-type three-level atom is sensitive to the mutual orientation of the atomic dipole moments. And SGC is responsible for many important physical phenomena involving potential application [29]-[36] in lasing without population inversion, coherent population trapping (CPT), group velocity reduction, ultra fast all-optical switching and transparent high-index materials, high-precision spectroscopy and magnetometer and modified quantum beats, etc. Inspired by these studies, we here utilize this quantum interference to explore an efficient scheme of 2D atom localization in a three-level $\Lambda$-type system. When the parameters corresponding to the standing-wave fields are changed, finding an atom is sensitive to the SGC intensities at a particular position within a wavelength domain. And a better resolution for 2D atom localization can be theoretically achieved in our scheme.

\section{Model and Equations}

We consider a $\Lambda$-type system as shown in Figure 1. The excited state $|1\rangle$ is coupled to the lower levels $|2\rangle$ and $|3\rangle$ via a standing-wave field $E_{x, y}$ and a week probe field $E_{p} \cdot 2 \gamma_{1}$ and $2 \gamma_{2}$ are the spontaneous decay rates of the excited state $|1\rangle$ to the ground states $|2\rangle$ and $|3\rangle$. When the two lower levels $|2\rangle$ and $|3\rangle$ are closely spaced such that the two transitions to the excited state interact with the same vacuum mode, SGC can be present. The standing-wave field $E_{x, y}$ is the superposition of two orthogonal standing-wave fields, i.e., one is in the $x$ direction and the second is along $y$ direction [26] [37]. The Rabi frequency corresponding to the probe field $E_{p}$ is $\Omega_{p}=E_{p} \mu_{13} / 2 \hbar$, and the position dependent Rabi frequency corresponding to the standingwave field $E_{x, y}$ is $\Omega_{c}(x, y)=E_{x, y} \mu_{12} / 2 \hbar$ where $\mu_{13}$ and $\mu_{12}$ are the corresponding dipole matrix elements.

The position-dependent Rabi frequency $\Omega_{c}(x, y)$ corresponding to the field $E_{c}(x, y)$ which is the superposition of two standing-wave fields $E_{c}(x)$ and $E_{c}(y)$ is defined as [26]

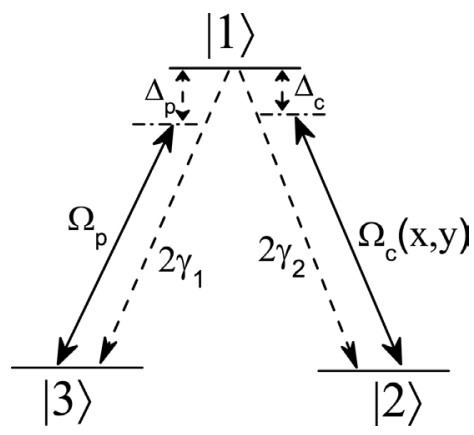

\footnotetext{
Figure 1. The position-dependent Rabi frequency $\Omega_{c}(x, y)$ corresponding to the atomic transition from $|1\rangle$ to $|2\rangle$ is due to the superposition of two standing wave fields, i.e., one is along the $\mathrm{x}$ direction and the second is along the y direction. The transition from $|1\rangle$ to $|3\rangle$ is coupled via a weak probe field $\Omega_{p}, 2 \gamma_{1}$ and $2 \gamma_{2}$ are the atomic decay rates.
} 


$$
\Omega_{c}(x, y)=\Omega_{0}\left[\sin \left(\kappa_{1} x+\delta\right)+\sin \left(\kappa_{2} y+\eta\right)\right]
$$

where $\kappa_{i}=2 \pi / \lambda_{i},(i=1,2)$ is the wave vector with wavelengths $\lambda_{i},(i=1,2)$ of the corresponding standing wave fields. The parameters $\delta$ and $\eta$ are the phase shifts associating with the standing-wave fields having wave vectors $\kappa_{1}$ and $\kappa_{2}$, respectively. We assume that the center-of-mass position of the atom along the direction of the standing-wave field is nearly constant. Therefore we neglect the kinetic-energy part of the Hamiltonian under the Raman-Nath approximation. In the interaction picture, Hamiltonian of this system in a rotating-wave frame is then given by

$$
H_{I}=\Delta_{c}|1\rangle\left\langle 1\left|+\left(\Delta_{c}-\Delta_{p}\right)\right| 3\right\rangle\langle 3|+\left[\Omega_{p}|1\rangle\left\langle 3\left|+\Omega_{c}\right| 1\right\rangle\langle 2|+\text { H.c. }\right] .
$$

Here, $\Delta_{c}=\omega_{12}-v_{c}$ and $\Delta_{p}=\omega_{13}-v_{p}$ are the field detunings corresponding to the atomic transitions $|1\rangle-$ $|2\rangle$ and $|1\rangle-|3\rangle$, respectively. $v_{c}$ and $v_{p}$ are the frequencies for the coupling standing-wave field and week probe field.

Under the rotating-wave approximation [38], the systematic density matrix in the interaction picture involving the SGC can be written as

$$
\begin{gathered}
\dot{\rho}_{11}=-2\left(\gamma_{1}+\gamma_{2}\right) \rho_{11}+i \Omega_{p} \rho_{31}+i \Omega_{c} \rho_{21}-i \Omega_{c}^{*} \rho_{12}-i \Omega_{p}^{*} \rho_{13}, \\
\dot{\rho}_{22}=2 \gamma_{2} \rho_{11}+i \Omega_{c}^{*} \rho_{12}-i \Omega_{c} \rho_{21} \\
\dot{\rho}_{33}=2 \gamma_{1} \rho_{11}+i \Omega_{p}^{*} \rho_{13}-i \Omega_{p} \rho_{31}, \\
\dot{\rho}_{12}=-\left(\gamma_{1}+\gamma_{2}+i \Delta_{c}\right) \rho_{12}+i \Omega_{p} \rho_{32}-i \Omega_{c}\left(\rho_{11}-\rho_{22}\right), \\
\dot{\rho}_{13}=-\left(\gamma_{1}+\gamma_{2}+i \Delta_{p}\right) \rho_{13}+i \Omega_{c} \rho_{23}-i \Omega_{p}\left(\rho_{11}-\rho_{33}\right), \\
\dot{\rho}_{23}=-i\left(\Delta_{p}-\Delta_{c}\right) \rho_{23}+2 p \sqrt{\gamma_{1} \gamma_{2}} \rho_{11}+i \Omega_{c}^{*} \rho_{13}-i \Omega_{p} \rho_{21},
\end{gathered}
$$

The above equations are constrained by $\rho_{11}+\rho_{22}+\rho_{33}=1$ and $\rho_{i j}^{*}=\rho_{j i}$. The effect of SGC is very sensitive to the orientations of the atomic dipole moments $\mu_{13}$ and $\mu_{12}$. Here, the parameter $p$ denotes the alignment of the two dipole moments and is defined as $p=\mu_{13} \cdot \mu_{12} /\left|\mu_{13} \cdot \mu_{12}\right|=\cos \theta$ with $\theta$ being the angle between the two dipole moments. So the parameter $p$ depicts the intensity of SGC in the atomic system. The terms with $p \sqrt{\gamma_{1} \gamma_{2}}$ represent the quantum interference resulting from the cross coupling between spontaneous emission paths $|1\rangle-|2\rangle$ and $|1\rangle-|3\rangle$. With the restriction of each field acting only on one transition, the Rabi frequencies $\Omega_{c}$ and $\Omega_{p}$ are connected to the angle $\theta$ and represented by $\Omega_{0}=\Omega_{c 0} \sin \theta, \Omega_{p}=\Omega_{p 0} \sin \theta$. It should be noted that only for small energy spacing between the two lower levels are the interference terms in the systematic density matrix significant; otherwise the oscillatory terms will average out to zero and thereby the SGC effect vanishes.

Our goal here is to obtain the information about the atomic position from the susceptibility of the system [14]-[16] at the probe field frequency. The nonlinear Raman susceptibility $\chi$ is then given by

$$
\chi=\frac{2 N\left|\mu_{13}\right|^{2}}{\varepsilon_{0} \Omega_{P} \hbar} \rho_{13}
$$

where $N$ is the atom number density in the medium and $\mu_{13}$ is the magnitude of the dipole-matrix element between $|1\rangle$ and $|3\rangle . \varepsilon_{0}$ is the permittivity in free space. For simplicity we assume $\Omega_{p}$ and $\Omega_{0}$ to be real. The general steady-state analytical solution for $\rho_{13}$ can be written as

$$
\rho_{13}=\frac{\Omega_{p} \Omega_{c}^{2}\left[B-A+\left(\Delta_{p}+2 i\right) C\right] C}{A^{2}(A-B)+(2 A-B) C\left(A-2 \Omega_{c}^{2}\right) \Delta_{c}+\left[\left(4+\Delta_{c}^{2}\right) A+\left(4 \Omega_{p}^{2}+B\right) \Omega_{c}^{2}-2 \Omega_{c}^{4}\right] C^{2}+\left(2 \Delta_{c}+C\right) \Omega_{c}^{2} C^{3}}
$$

with $A=\Omega_{p}^{2}+\Omega_{c}^{2}, B=2 \Omega_{c} p \cos \theta \cdot \Omega_{p}, C=\Delta_{p}-\Delta_{c}$, and we have set $\gamma_{1}=\gamma_{2}=\gamma$. All the parameters are reduced to dimensionless units by scaling with $\gamma$. Thus the linear susceptibility $\chi$ at the probe frequency can therefore be calculated using Equation (9), which consists of both real and imaginary parts, i.e., $\chi=\chi^{\prime}+i \chi^{\prime \prime}$. 
The imaginary part of the susceptibility gives the absorption profile of the probe field which can be written as

$$
\chi^{\prime \prime}=\frac{2 N\left|\mu_{13}\right|^{2}}{\epsilon_{0} \hbar} \operatorname{Im}\left[\frac{\rho_{13}}{\Omega_{P}}\right]=\alpha \operatorname{Im}\left[\frac{\rho_{13}}{\Omega_{P}}\right],
$$

where $\alpha=\frac{2 N\left|\mu_{13}\right|^{2}}{\epsilon_{0} \hbar}$. Here we are interested in the precise position measurement of the atom using the absorption process of the probe field. Equation (11) is the main result and reflects the position probability distribution of the atom [14] [15]. It can be seen that the probe absorption depends on the position dependent SGC intensities, therefore, we can obtain the position information of the atom by measuring the probe absorption.

\section{Results and Discussion}

The schematic our considered in Figure 1 can be understood more clearly when the combination of standing-wave fields with the corresponding position-dependent Rabi frequency $\Omega_{c}(x, y)$ is replaced by a simple driving field with the corresponding Rabi frequency $\Omega_{c}$ [39]. As mentioned earlier [14]-[16] [26], it is clear that the expression (11), which exhibits the probe field absorption, depends on the controllable parameters like the intensities and phase shifts of the standing-wave fields, the detunings of the probe field. Our aim is to investigate the precise location of the 2D atom localization via $\chi^{\prime \prime}$. Here, we consider different controllable parameters for the atomic position localization, i.e., the interference between spontaneous emission channels, i.e., SGC. The expression (11) reflecting the atomic position probability distribution associating with the intensities p of SGC is rather cumbersome. Hence, we follow the numerical approach and analyze the position probability distribution via $\chi^{\prime \prime}$.

Initially, we set the detuning $\Delta_{c}=0, \Delta_{p}=15 \gamma$, the phase shifts associating with standing-wave fields $\delta=\eta=0$, and the Rabi frequency $\Omega_{c 0}=10 \gamma, \Omega_{p 0}=0.3 \gamma$. The wavelengths' parameters were set $\kappa_{1}=\kappa_{2}=\pi / 4$, which means wavelengths for the standing-wave fields are 8 wavelength units. For these choices of parameters, we consider the atomic position probability distribution dependent different intensities of SGC (depicted by $p$ ).

The position-dependent the intensities of SGC is shown in Figure 2, in which $\chi^{\prime \prime}$ is plotted versus position $x$ and $y$ within the optical wavelength. The intensities of SGC are Figure 2 (a) $p=0.87$, (b) $p=0.81$, (c) $p=$ 0.71 , (d) $p=0.50$. Two spike-like localization peaks sit in the second and fourth quadrants of the $x$-y plane, and their same amplitudes are about 0.02 in Figure 2(a) when $p=0.92$. The amplitude of the position probability distribution increases to 0.04 when the intensities of SGC was tuned to 0.81 in Figure 2(b). The double spikelike peaks become more sharp and their peak values reach 0.3 in Figure 2(c) for $p=0.71$. However, two craterlike structures show in the second and fourth quadrants of the $\mathrm{x}-\mathrm{y}$ plane when $p=0.50$ in Figure 2(d), in spite of the amplitude of the localization peak increasing considerably. Under these setting parameters, the best resolution for the 2D atomic localization is obtained when the intensity of SGC with $p=0.71$ in Figure 2(c), and the resolution becomes ambiguous when $p=0.5$ in Figure 2(d).

We next study how the detuning associating with standing-wave fields brings changes in the $2 \mathrm{D}$ atomic localization, i.e, $\Delta_{c}=-1.5 \gamma$. In Figure 3, $\chi^{\prime \prime}$ was plotted versus position $x$ and $y$ with other parameters being the same as those in Figure 2. The decreasing intensities of SGC are equal to Figure 3 (a) $p=0.92$, (b) $p=0.87$, (c) $p=0.81$, and (d) $p=0.79$. The results presented in Figure 3 from Figure 3(a) to Figure 3(d) are gratifying. The dual spike-like peaks with increasing amplitudes are shown in Figure 3 from Figure 3(a) to Figure 3(d), and their values are $0.015,0.03,0.08,0.10$, respectively. We noted that the increasing resolution for $2 \mathrm{D}$ atomic localization can be obtained when the standing-wave fields couples the transition $|1\rangle \leftrightarrow|2\rangle$ off-resonantly.

After studying the 2D atomic localization dependent the detuning associating with standing-wave fields, we further study how the phase shifts $\delta, \eta$ associating with standing-wave fields bring changes in the 2D atomic localization. Due to the periodicity associated with the position-dependent Rabi frequency $\Omega_{c}(x, y)$ ), there will be more than one position probability distribution for the $2 \mathrm{D}$ atom localization in the $\mathrm{x}$-y plane. The multiple peaks are much more ambiguous than the unique peak for 2D atom localization. We look for the roles of phases $\delta$ and $\eta$ associated with the standing-wave fields in the 2D atomic localization. In Figure 4, the density plots of the 2D position probability distribution are shown for different intensities of SGC, i.e., (a) $p=0.5$, (b) $p=$ 0.71 , (c) $p=0.81$, and we set $\delta=\eta=\pi / 2, \kappa_{1}=\kappa_{2}=\pi / 8, \Delta_{p}=12 \gamma$, other parameters are the same as those 

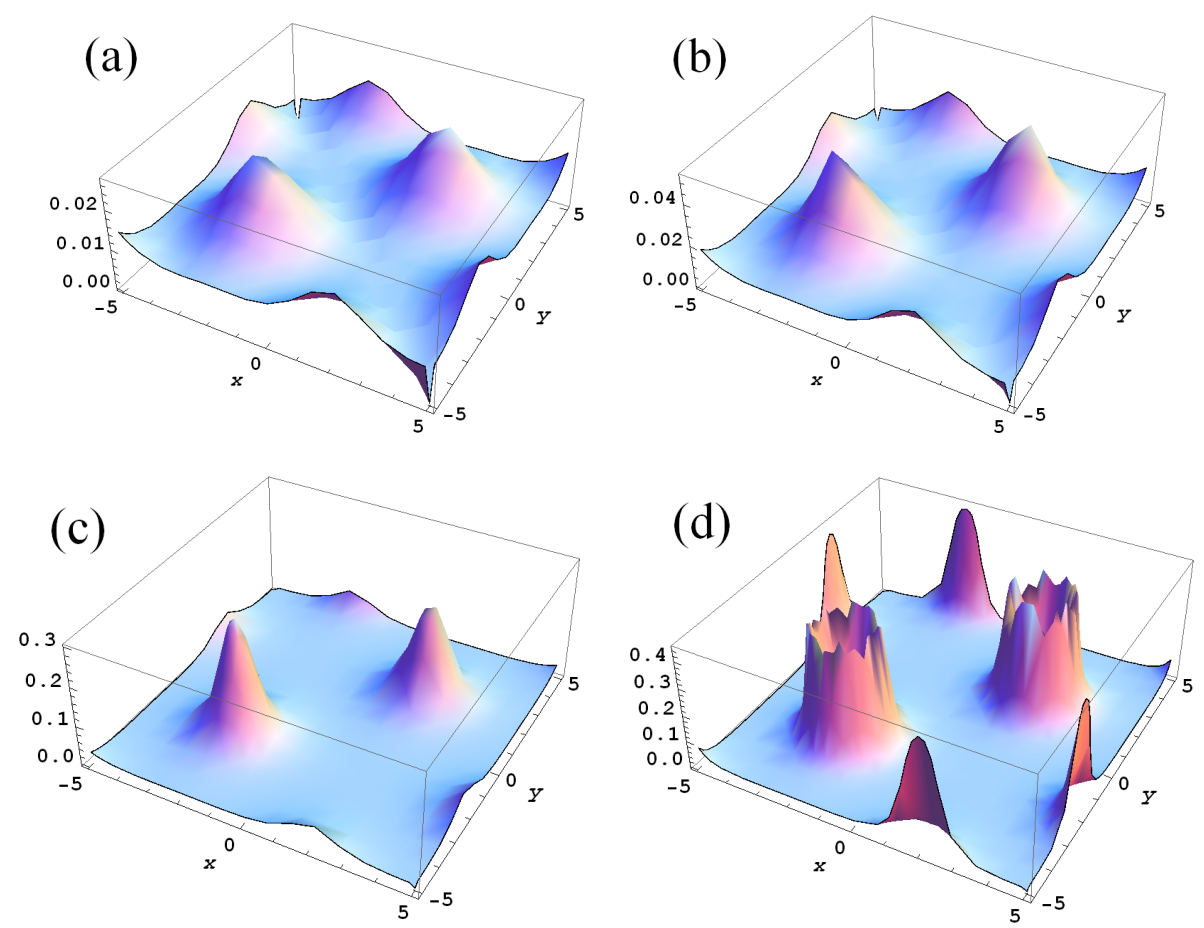

Figure 2. (Color online) Plots for 2D atom localization: $\chi^{\prime \prime}$ versus the positions $x$ and $y$ for different intensities p of SGC. (a) $p=0.87$, (b) $p=0.81$, (c) $p=0.71$, (d) $p=0.50$. Other parameters are $\Delta_{p}=15 \gamma, \Delta_{c}=0, \alpha=\gamma$, and $\gamma_{1}=\gamma_{2}=\gamma$ where $\gamma$ is the scaling parameter.
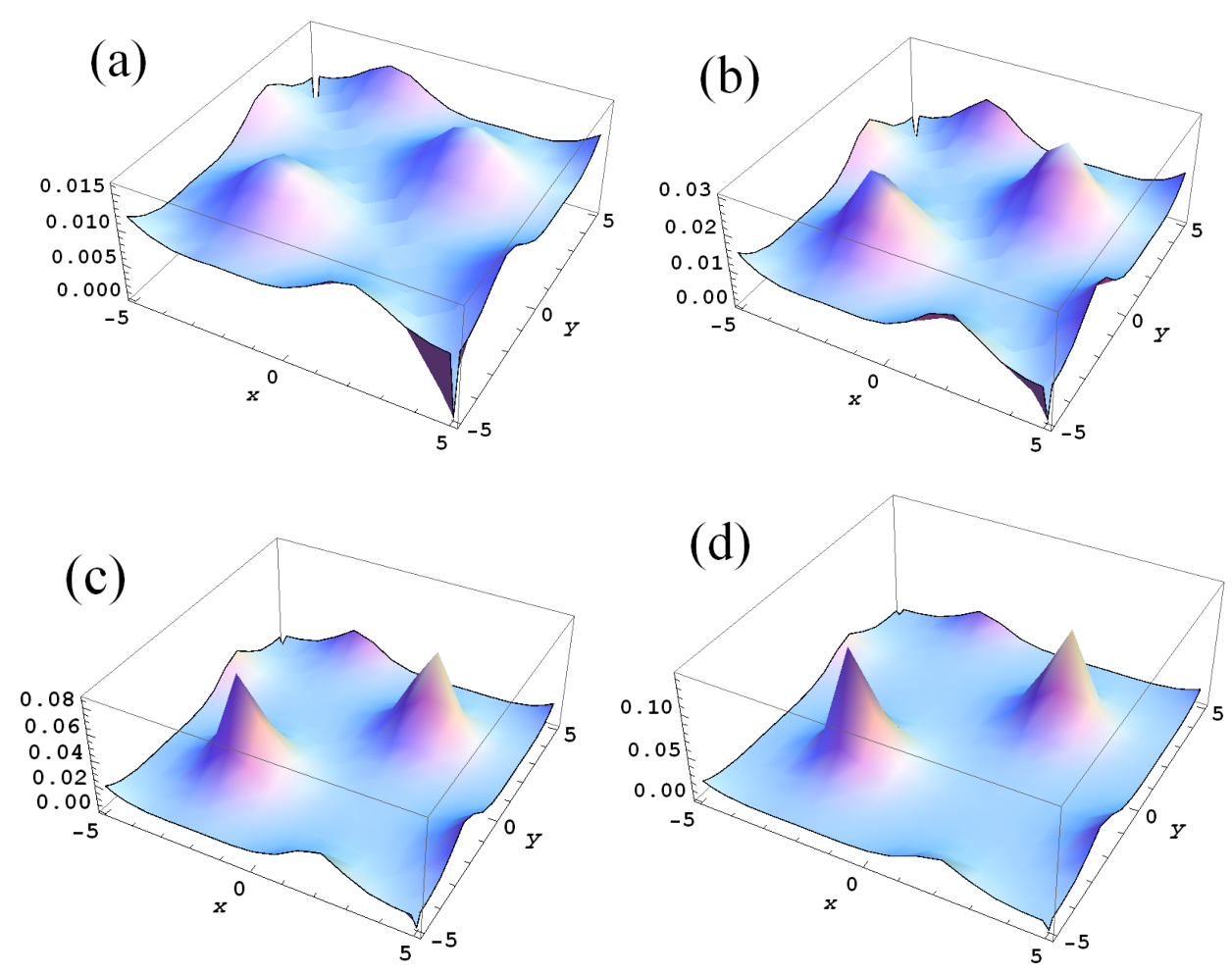

Figure 3. (Color online) Plots for 2D atom localization: $\chi^{\prime \prime}$ versus the positions $x$ and $y$ for different intensities p of SGC. (a) $p=0.92$, (b) $p=0.87$, (c) $p=0.81$, (d) $p=0.79, \Delta_{c}=-1.5 \gamma$. The other parameters are the same as in Figure 2. 

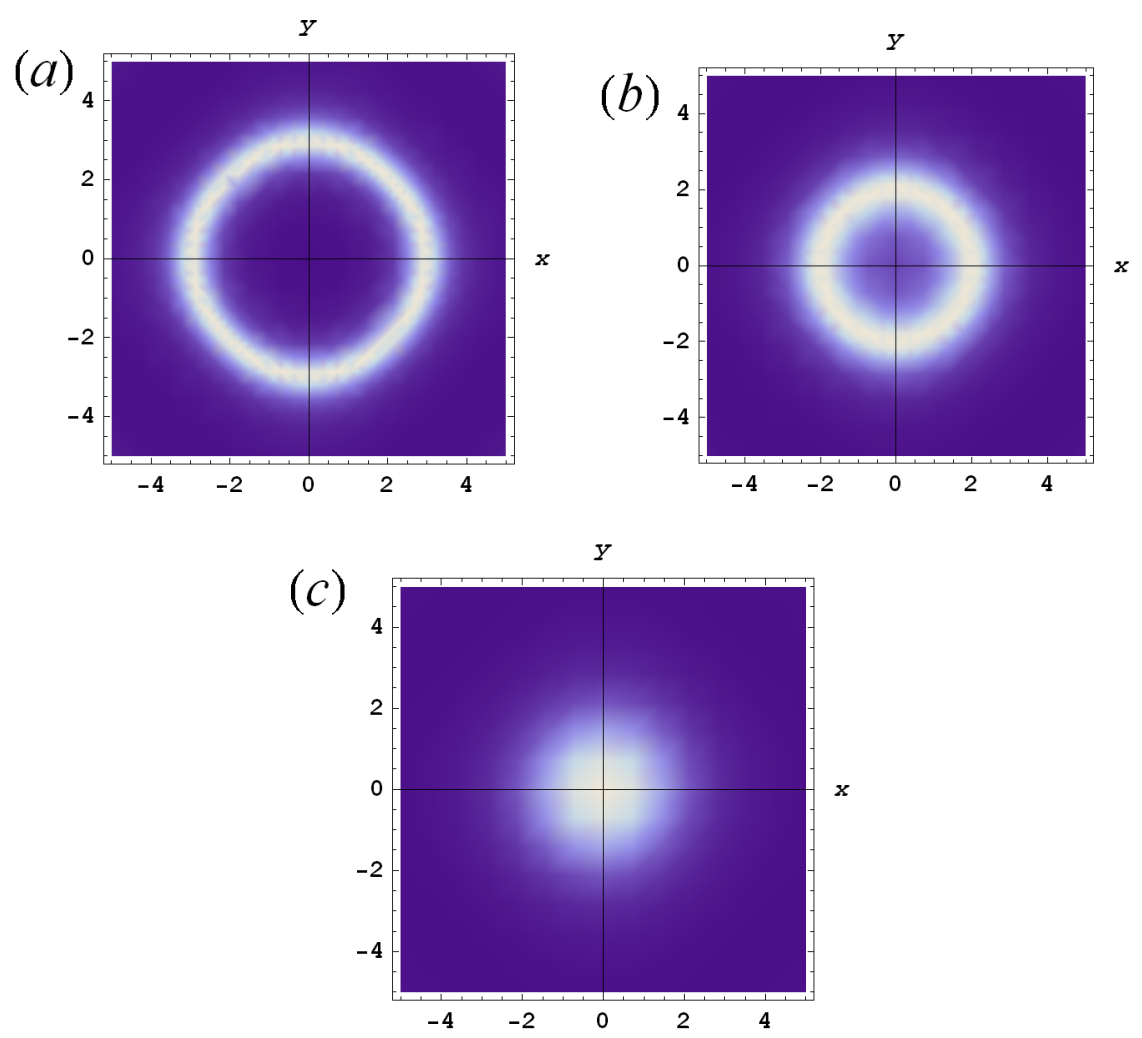

Figure 4. (Color online) Density plots of 2D atom localization: Plots of $\chi^{\prime \prime}$ for different intensities $p$ of SGC. (a) $p=0.5$, (b) $p=0.71$, (c) $p=0.81$. $\kappa_{1}$ and $\kappa_{2}=\pi / 8$, $\delta=\eta=\pi / 2, \Delta_{p}=12 \gamma$. All the other parameters are the same as in Figure 2.

\section{Conclusion}

On the basis of a three-level $\Lambda$-type atom model, we investigated its 2D atom localization via different parameters of the two orthogonal standing-wave fields with the decreasing or increasing intensities of SGC. Two spikelike peaks with flexible amplitudes for the atom localization are shown in the $\mathrm{x}-\mathrm{y}$ plane by the decreasing intensities of SGC when the detuning corresponding to the two orthogonal standing-wave fields is varied, while the increasing intensities of SGC reduce the resolution of 2D atom localization strongly. When the phases and wave vectors corresponding to the standing-wave fields are changed, one circular ring with shrinking radii for 2D atom localization is obtained by the increasing intensities of SGC. Comparing the phases and wave vectors with the detuning corresponding to the two orthogonal standing-wave fields, a better resolution can be obtained by the phases and wave vectors corresponding to the two orthogonal standing-wave fields when SGC was manipulated. Considering the proposed three-level atom-field system to be a simple system which would be realized experimentally, such as the bichromatic EIT in cold rubidium atoms $\left({ }^{87} \mathrm{Rb}\right)$, our scheme and results may be of great interest for the researchers.

\section{Fund}

This paper is supported by the National Natural Science Foundation of China (Grant Nos. 61205205 and 6156508508), the General Program of Yunnan Provincial Research Foundation of Basic Research for application (Grant No. 2016FB009) and the Foundation for Personnel training projects of Yunnan Province, China (Grant No. KKSY201207068).

\section{References}

[1] Storey, P. and Collett, M. (1993) Atomic-Position Resolution by Quadrature-Field Measurement. Physical Review A, 
47, 405-418. http://dx.doi.org/10.1103/PhysRevA.47.405

[2] Phillips, W.D. (1998) Laser Cooling and Trapping of Neutral Atoms. Reviews of Modern Physics, 70, 721-741. http://dx.doi.org/10.1103/RevModPhys.70.721

[3] Johnson, K.S., Thywissen, J.H., Dekker, W.H., Berggren, K.K., Chu, A.P., Younkin, R. and Prentiss, M. (1998) Localization of Metastable Atom Beams with Optical Standing Waves: Nanolithography at the Heisenberg Limit. Science, 280, 1583-1586. http://dx.doi.org/10.1126/science.280.5369.1583

[4] Collins, G.P. (1996) Experimenters Produce New Bose-Einstein Condensate(s) and Possible Puzzles for Theorists. Phys. Today, 49, 18-21. http://dx.doi.org/10.1063/1.2807533

[5] Kapale, K.T., Qamar, S. and Zubairy, M.S. (2003) Spectroscopic Measurement of an Atomic Wave Function. Physical Review A, 67, 023805. http://dx.doi.org/10.1103/PhysRevA.67.023805

[6] Adams, C.S., Sigel, M. and Mlynek, J. (1994) Atom Optics. Physics Reports, 240, 143-210. http://dx.doi.org/10.1016/0370-1573(94)90066-3

[7] Storey, P., Collett, M. and Walls, D. (1992) Measurement-Induced Diffraction and Interference of Atoms. Physical Review Letters, 68, 472. http://dx.doi.org/10.1103/PhysRevLett.68.472

[8] Marte, M.A.M. and Zoller, P. (1992) Quantum Nondemolition Measurement of Transverse Atomic Position in KapitzaDirac Atomic Beam Scattering. Applied Physics B, 54, 477-485. http://dx.doi.org/10.1007/BF00325394

[9] Quadt, R., Collett, M. and Walls, D.F. (1995) Measurement of Atomic Motion in a Standing Light Field by Homodyne Detection. Physical Review Letters, 74, 351. http://dx.doi.org/10.1103/PhysRevLett.74.351

[10] Carmichael, H.J. (1993) An Open Systems Approach to Quantum Optics: Lecture Notes in Physics. Springer-Verlag, Berlin.

[11] Evers, J., Qamar, S. and Zubairy, M.S. (2007) Atom Localization and Center-of-Mass Wave-Function Determination via Multiple Simultaneous Quadrature Measurements. Physical Review A, 75, Article ID: 053809. http://dx.doi.org/10.1103/PhysRevA.75.053809

[12] Paspalakis, E. and Knight, P.L. (2001) Localizing an Atom via Quantum Interference. Physical Review A, 63, Article ID: 065802. http://dx.doi.org/10.1103/PhysRevA.63.065802

[13] Paspalakis, E., Terzis, A.F. and Knight, P.L. (2005) Quantum Interference Induced Sub-Wavelength Atomic Localization. Journal of Modern Optics, 52, 1685-1694. http://dx.doi.org/10.1080/09500340500072489

[14] Sahrai, M., Tajalli, H., Kapale, K.T. and Zubairy, M.S. (2005) Subwavelength Atom Localization via Amplitude and Phase Control of the Absorption Spectrum. Physical Review A, 72, Article ID: 013820. http://dx.doi.org/10.1103/PhysRevA.72.013820

[15] Kapale, K.T. and Zubairy, M.S. (2006) Subwavelength Atom Localization via Amplitude and Phase Control of the Absorption Spectrum. II. Physical Review A, 73, Article ID: 023813. http://dx.doi.org/10.1103/PhysRevA.73.023813

[16] Qamar, S., Mehmood, A. and Qamar, S. (2009) Subwavelength Atom Localization via Coherent Manipulation of the Raman Gain Process. Physical Review A, 79, Article ID: 033848. http://dx.doi.org/10.1103/physreva.79.033848

[17] Qamar, S., Evers, J. and Zubairy, M.S. (2009) Atom Microscopy via Two-Photon Spontaneous Emission Spectroscopy. Physical Review A, 79, Article ID: 043814. http://dx.doi.org/10.1103/physreva.79.043814

[18] Wan, R.G., Kou, J., Jiang, L., Jiang, Y. and Gao, J.Y. (2011) Two-Dimensional Atom Localization via Interacting Double-Dark Resonances. Journal of the Optical Society of America B, 28, 10-17. http://dx.doi.org/10.1364/JOSAB.28.000010

[19] Ghafoor, F. (2011) Subwavelength Atom Localization via Quantum Coherence in a Three-Level Atomic System. Physical Review A, 84, Article ID: 063849. http://dx.doi.org/10.1103/physreva.84.063849

[20] Rahmatullah, Z. and Khan, A. (2012) Precision Position Measurement of Single Atom. Chinese Optics Letters, 10, 7578.

[21] Asghar, S. and Qamar, S. (2013) Atom Microscopy Using Time-Dependent Physical Spectrum. Optics Communications, 295, 145-149. http://dx.doi.org/10.1016/j.optcom.2012.12.075

[22] Ivanov, V. and Rozhdestvensky, Y. (2010) Two-Dimensional Atom Localization in a Four-Level Tripod System in Laser Fields. Physical Review A, 81, Article ID: 033809. http://dx.doi.org/10.1103/physreva.81.033809

[23] Wan, R.G., Kou, J., Jiang, L., Jiang, Y. and Gao, J.Y. (2011) Two-Dimensional Atom Localization via Quantum Interference in a Coherently Driven Inverted-Y System. Optics Communications, 284, 985-990. http://dx.doi.org/10.1016/j.optcom.2010.10.066

[24] Ding, C., Li, J., Zhan, Z. and Yang, X. (2011) Two-Dimensional Atom Localization via Spontaneous Emission in a Coherently Driven Five-Level M-Type Atomic System. Physical Review A, 83, Article ID: 063834. http://dx.doi.org/10.1103/physreva.83.063834 
[25] Ding, C., Li, J., Yang, X., Zhang, D. and Xiong, H. (2011) Proposal for Efficient Two-Dimensional Atom Localization Using Probe Absorption in a Microwave-Driven Four-Level Atomic System. Physical Review A, 84, Article ID: 043840. http://dx.doi.org/10.1103/physreva.84.043840

[26] Ullah, R. and Qamar, S. (2013) Two-Dimensional Atom Localization via Probe-Absorption Spectrum. Physical Review A, 88, Article ID: 013846. http://dx.doi.org/10.1103/physreva.88.013846

[27] Javanainen, J. (1992) Effect of State Superpositions Created by Spontaneous Emission on Laser-Driven Transitions. Europhysics Letters, 17, 407-411. http://dx.doi.org/10.1209/0295-5075/17/5/005

[28] Höler, G. (1974) Tracts in Modern Physics. Springer, Berlin, 70.

[29] Harris, S.E. (1989) Lasers without Inversion: Interference of Lifetime-Broadened Resonances. Physical Review Letters, 62, 1033-1036. http://dx.doi.org/10.1103/PhysRevLett.62.1033

[30] Zhu, S.Y. and Scully, M.O. (1996) Spectral Line Elimination and Spontaneous Emission Cancellation via Quantum Interference. Physical Review Letters, 76, 388-391. http://dx.doi.org/10.1103/PhysRevLett.76.388

[31] Paspalakis, E., Kylstra, N.J. and Knight, P.L. (2000) Transparency of a Short Laser Pulse via Decay Interference in a Closed V-Type System. Physical Review A, 61, Article ID: 045802. http://dx.doi.org/10.1103/PhysRevA.61.045802

[32] Wu, J.H., Gao, J.Y. and Xu, J.H. (2005) Ultrafast All Optical Switching via Tunable Fano Interference. Physical Review Letters, 95, Article ID: 057401. http://dx.doi.org/10.1103/physrevlett.95.057401

[33] Hong, T., Cramer, C., Nagourney, W. and Fortson, E.N. (2005) Optical Clocks Based on Ultranarrow Three-Photon Resonances in Alkaline Earth Atoms. Physical Review Letters, 94, Article ID: 050801. http://dx.doi.org/10.1103/physrevlett.94.050801

[34] Joshi, A. and Xiao, M. (2005) Dark-State Polaritons Using Spontaneously Generated Coherence. European Physical Journal D, 35, 547-551. http://dx.doi.org/10.1140/epjd/e2005-00093-9

[35] Paternostro, M., Kim, M.S. and Knight, P.L. (2005) Vibrational Coherent Quantum Computation. Physical Review A, 71, Article ID: 022311. http://dx.doi.org/10.1103/physreva.71.022311

[36] Dutta, B.K., Panchadhyayee, P. and Mahapatra, P.K. (2013) Coherent Control of Localization of a Three-Level Atom by Symmetric and Asymmetric Superpositions of Two Standing-Wave Fields. Laser Physics, 23, Article ID: 045201.

[37] Dutta, B.K., Panchadhyayee, P. and Mahapatra, P.K. (2012) Precise Localization of a Two-Level Atom by the Superposition of Two Standing-Wave Fields. Journal of the Optical Society of America B, 29, 3299-3306. http://dx.doi.org/10.1364/JOSAB.29.003299

[38] Scully, M.O. and Zubairy, M.S. (1997) Quantum Optics. Cambridge University Press, Cambridge. http://dx.doi.org/10.1017/CBO9780511813993

[39] Wang, J., Zhu, Y., Jiang, K.J. and Zhan, M.S. (2003) Bichromatic Electromagnetically Induced Transparency in Cold Rubidium Atoms. Physical Review A, 68, Article ID: 063810. http://dx.doi.org/10.1103/physreva.68.063810

\section{Submit or recommend next manuscript to SCIRP and we will provide best service for you:}

Accepting pre-submission inquiries through Email, Facebook, LinkedIn, Twitter, etc.

A wide selection of journals (inclusive of 9 subjects, more than 200 journals)

Providing 24-hour high-quality service

User-friendly online submission system

Fair and swift peer-review system

Efficient typesetting and proofreading procedure

Display of the result of downloads and visits, as well as the number of cited articles

Maximum dissemination of your research work

Submit your manuscript at: http://papersubmission.scirp.org/ 